\title{
Eficácia das Vacinas Contra o Vírus do Papiloma Humano nas Mulheres com mais de 24 Anos na Prevenção do Cancro do Colo do Útero
}

\author{
Efficacy of the Vaccines Against Human Papillomavirus in \\ Women Older than 24 Years in the Cervix Cancer Prevention
}

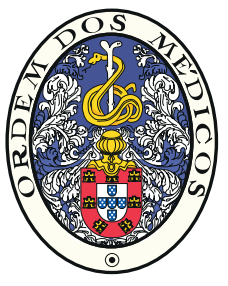

Jaime Pimenta RIBEIRO $\mathbb{1}^{1}$, Isabel BORGES 1

Acta Med Port 2016 Jun;29(6):401-408 - http://dx.doi.org/10.20344/amp.7095

RESUMO

Introdução: O cancro do colo do útero é o quarto tipo de cancro feminino mais comum e o segundo mais frequente nas mulheres entre os 15 e os 44 anos. A infeção pelo vírus do papiloma humano é condição necessária na quase totalidade dos casos de cancro do colo do útero; trata-se de uma doença sexualmente transmissível e todas as mulheres sexualmente ativas estão em risco de contrair infeção. Atualmente existem três vacinas contra o vírus do papiloma humano, e apesar da sua eficácia estar bem comprovada nas mulheres até aos 25 anos, não está totalmente estabelecida em idades superiores.

Material e Métodos: Realizou-se uma revisão da literatura sobre a evidência atual relativa à eficácia das vacinas contra o vírus do papiloma humano nas mulheres com mais de 24 anos na prevenção do cancro do colo do útero e foi avaliada a qualidade da evidência segundo o sistema GRADE.

Resultados: Foram identificados dois estudos controlados randomizados relevantes para a análise que envolveram um total de 9571 participantes - 4792 no grupo vacinado e 4779 no grupo controlo - e reportaram dados relativos a 48 meses de follow-up. A redução do risco relativo foi de $41 \%$ (95\% Cl: 29 a 50\%), - favorável para o grupo vacinado. Não existiram diferenças entre a segurança e tolerabilidade das vacinas nos grupos vacina e controlo.

Conclusão: A evidência atual demonstra que as vacinas contra o vírus do papiloma humano 16 e/ou 18 nas mulheres com mais de 24 e até aos 45 anos apresentam uma eficácia importante na redução do risco de desenvolver infeção persistente e/ou adquirir lesões precursoras de cancro do colo do útero.

Palavras-chave: Adulto; Feminino; Infecções por Papillomavirus/prevenção e controlo; Neoplasias do Colo do Útero/prevenção e controlo; Vacinas contra Papillomavirus; Vacinação.

\section{ABSTRACT}

Introduction: Cervix cancer is the fourth most common female cancer and the second most frequent in women aged 15 to 44 years. The infection by the human papillomavirus is a necessary condition in almost all of cervix cancer cases; it is a sexually transmitted disease and all women sexually active are at risk of being infected. There are currently three vaccines against human papillomavirus, and despite of the efficacy being well proven in woman aged up to 25 years, it is not well established in older ages.

Material and Methods: It was performed a literature review of the current evidence about the efficacy of the vaccines against human papillomavirus in women older than 24 years in the cervix cancer prevention, and was assessed the evidence quality by the GRADE system.

Results: Two randomized controlled trials were identified with a total of 9,571 participants $-4,792$ vaccinated and 4,779 at the control group and reported data of 48 months period follow-up. The relative risk reduction was $41 \%$ (95\% Cl: 29 a $50 \%)$ - for the vaccinated group. There were no differences in safety and tolerability between the two groups - vaccine and control.

Conclusion: The actual evidence shows that the vaccines against human papillomavirus 16 and/or 18 in women older than 24 until 45 years have an important efficacy at reducing the risk of having persistent infection and/or to acquire cervix cancer precursor lesions.

Keywords: Adult; Female; Papillomavirus Infections/prevention \& control; Papillomavirus Vaccines; Uterine Cervical Neoplasms/ prevention \& control; Vaccination.

\section{INTRODUÇÃO}

O cancro do colo do útero (CCU) é o quarto tipo de cancro mais frequente no sexo feminino ${ }^{1-3}$ e representa $o$ segundo mais comum nas mulheres com idade entre os 15 e 44 anos. $^{2}$ Calcula-se que em 2012 tenham ocorrido aproximadamente 528000 novos casos de CCU e 266000 mortes relacionadas com a patologia. ${ }^{2,3}$

Em Portugal, apesar da existência de um programa nacional de rastreio e da introdução da vacinação contra o vírus do papiloma humano (HPV) no plano nacional de vacinação em 2008, o CCU representa ainda o sétimo tipo de cancro feminino mais frequente e o segundo mais comum na mulher entre 15 e 44 anos. ${ }^{4}$ Entre os anos 2000 e 2013 morreram mais de 200 mulheres por ano devido ao CCU (cerca de três em cada 100000 mulheres), ${ }^{5}$ no entanto, este valor poderá representar uma subestimativa, uma vez que alguns casos de CCU são classificados como "tumor maligno do útero, porção não especificada" e não são contabilizados nesta estatística. ${ }^{5}$

A infeção pelo HPV é condição necessária na quase totalidade dos casos de CCU, ${ }^{5-10}$ existindo mais de 100 genótipos do HPV estudados; $90 \%$ dos casos de CCU são provocados pelos genótipos de alto risco (oncogénicos) 16 , $18,45,31,33,52,58$ e 35.,10-14 A infeção pelos genótipos 16 e 18 está na origem da grande maioria dos casos de CCU

\footnotetext{
1. Medicina Geral e Familiar. Unidade de Saúde Familiar Corgo. Vila Real. Portugal.

$\square$ Autor correspondente: Jaime Pimenta Ribeiro. jaime.pim.ribeiro@gmail.com

Recebido: 15 de outubro de 2015 - Aceite: 27 de abril de 2016 | Copyright @ Ordem dos Médicos 2016
} 
- mais de $70 \%$ dos casos, $, 5,6,10,14-17$ enquanto aproximadamente $10 \%$ são consequência da infeção pelos genótipos 45 e $31,{ }^{15}$ e os restantes são causados por outros genótipos oncogénicos. Esta infeção é uma doença sexualmente transmissível, representando uma das mais comuns, ${ }^{11,12,14}$ o genótipo 16 é o mais prevalente, ${ }^{12}$ apesar de existir variação geográfica na prevalência dos genótipos..$^{11,18}$ Todas as mulheres sexualmente ativas estão em risco de contrair infeção por genótipos do HPV oncogénicos, estimando-se que até $80 \%$ das mulheres sofram infeção por HPV (genótipos oncogénicos e/ou não-oncogénicos) ao longo da vida, ${ }^{9,15}$ sendo que pelo menos $50 \%$ serão infetadas dois a cinco anos logo após o início da atividade sexual, permanecendo, contudo, o risco de infeção significativo em idades mais avançadas. ${ }^{18,19}$ A maioria das infeções por HPV é transitória, ${ }^{6,15}$ ocorrendo resolução espontânea em $90 \%$ dos casos após dois anos, ${ }^{15}$ que, no entanto, não confere imunidade contra a infeção pelo vírus. ${ }^{6,9,12,13,15,16}$

A eficácia das vacinas contra o HPV nas mulheres até aos 25 anos de idade está bem comprovada, ${ }^{10,11,16,17,19}$ porém, a eficácia em idades superiores ainda não está totalmente estabelecida e existem dúvidas quanto à sua utilização.

Atualmente existem três vacinas contra o HPV disponíveis: a tetravalente; a bivalente; e a nonavalente. A vacina tetravalente protege contra os genótipos do HPV 16, 18, 6 e 11 tendo sido aprovada em 2006, enquanto a vacina bivalente protege contra os genótipos 16 e 18 e foi aprovada em $2007,5,11,12,20$ a vacina nonavalente protege contra os genótipos $16,18,45,31,33,52,58,6$ e $11^{111,14}$ e foi aprovada recentemente, nos EUA em $2014^{21}$ e na Europa em $2015^{22}$. Estas vacinas possuem apenas carácter profilático e não se trata de vacinas terapêuticas, , $^{6}$ 9,11,12, 14,15,17 não sendo por isso capazes de erradicar a infeção uma vez estabelecida; contudo são capazes de conferir proteção contra os genótipos da vacina para os quais a mulher não apresente infeção e de evitar a reinfeção na mulher que resolveu espontaneamente a infeção prévia por genótipos presentes na vacina. ${ }^{9,11,12,15}$ As vacinas bivalente e tetravalente possuem um benefício comparável na prevenção do CCU, sendo capazes de prevenir pelo menos até $70 \%$ dos casos, ${ }^{6,10,12,14,15}$ enquanto, que a vacina nonavalente, teoricamente, será capaz de prevenir até $90 \%$ dos casos. ${ }^{11} \mathrm{Com}$ recurso a modelos matemáticos (resultantes de estudos de imunogenicidade), é possível prever que a duração da proteção conferida pelas vacinas seja superior a 30 anos. ${ }^{6,14,15}$ A segurança e tolerabilidade das vacinas foi avaliada em vários estudos, apresentando perfis semelhantes nos grupos vacinados e controlo, e foram consideradas vacinas com alto perfil de segurança, ${ }^{6,9,12,15,16,20,23}$ é ainda necessário comprovar este facto para a vacina nonavalente, devido à sua recente aprovação e à existência de poucos estudos.

\section{Objetivos}

Dadas as dúvidas quanto à eficácia e utilização das vacinas contra o HPV nas mulheres com mais de 24 anos, com este estudo pretende-se averiguar a sua eficácia nas mulheres com mais de 24 anos na prevenção do CCU, através de uma revisão da literatura sobre a evidência atual e avaliar a qualidade da evidência.

\section{MATERIAL E MÉTODOS}

Esta revisão de literatura foi realizada de acordo com as guidelines PRISMA ${ }^{24}$ para revisões sistemáticas (PRISMA checklist em informação suplementar).

\section{Critérios de elegibilidade}

Formulámos uma questão do tipo PICO (população, intervenção, controlo, outcome) (Tabela 1) que definiu os principais critérios de seleção e inclusão dos estudos. Considerámos elegíveis estudos realizados em qualquer país, publicados ou apenas registados em ClinicalTrials.gov (ainda não publicados), desde 1995 até agosto de 2015, escritos na língua inglesa ou portuguesa. Apenas foram elegíveis estudos controlados randomizados (RCTs), com baixo risco de viés, com resultados finais ou intermédios dos estudos, e com tempo de follow-up mínimo de quatro anos (48 meses).

\section{Fonte de informação}

Procedemos à pesquisa de literatura nas bases de dados Pubmed, The Cochrane Library, ClinicalKey, ClinicalTrials.gov, Index das Revistas Médicas Portuguesas, e ainda no site da Direção Geral de Saúde (DGS), com abrangência do intervalo de tempo de 1995 a agosto de 2015.

A pesquisa de literatura foi executada pelos dois investigadores em conjunto e decorreu em agosto de 2015; usámos os termos e os operadores de pesquisa descritos na Tabela 2.

\section{Seleção dos estudos}

Todas as citações identificadas foram analisadas pelos dois investigadores, selecionando as potencialmente relevantes; cada investigador, de forma independente, analisou o resumo das citações identificadas e avaliou o texto integral das publicações que considerou relevantes, tendo construído uma grelha para comparação e discussão. Selecionámos os estudos que cumpriram os critérios de elegibilidade descritos.

Tabela 1 - Critérios de inclusão na revisão sistemática - questão do tipo PICO.

\begin{tabular}{ll}
\hline População & Mulheres com idade igual ou superior a 24 anos \\
Intervenção & Vacinação contra o HPV, independentemente da vacina utilizada \\
Controlo & Placebo ou outra vacina que não a vacina contra o HPV ou não vacinação \\
Outcome & Infeção persistente por HPV 16 e/ou 18 \\
& Lesões do colo do útero provocadas pela infeção persistente por HPV
\end{tabular}


Tabela 2 - Termos usados na pesquisa da literatura

\begin{tabular}{ll}
\hline Termos em inglês & Termos em português \\
\hline \# HPV & \# vírus do papiloma humano \\
\# papillomavirus & \# vacinação \\
\# papillomaviridae & \# vacina(s) \\
\# vaccination & \# mulher adulta \\
\# vaccine(s) & \# cancro do colo do útero \\
\# adult women & \# cancro cervical \\
\# cervical cancer & \# E \# OU \\
\# AND \# OR &
\end{tabular}

\section{Definição de variáveis}

Definimos as principais variáveis do estudo na questão do tipo PICO (Tabela 1).

Estabelecemos que o limite superior de idade a usar na análise seria o mais alargado possível e que permitisse comparar os estudos; definiu-se o limite superior de idade de 45 anos, pois apenas em um dos estudos participou um pequeno grupo de mulheres com idade superior, não permitindo fazer comparações nem retirar conclusões válidas.

Para a variável outcome, assumimos: a duração da infeção por HPV 16 e/ou 18 definida nos estudos como sendo infeção persistente - igual ou superior a seis meses; e as lesões definidas nos estudos como sendo lesões provocadas pela infeção persistente por HPV 16 e/ou 18 - neoplasia intra-epitelial cervical (CIN) 1 ou superior.

\section{Avaliação do risco de viés}

Avaliámos o risco de viés com recurso à ferramenta de avaliação da Cochrane; ${ }^{25}$ foi tido em conta o método de geração da sequência aleatória, a ocultação da alocação, o mascaramento dos participantes e dos profissionais, o mascaramento dos avaliadores dos outcomes, dados dos outcomes incompletos, e o relato seletivo. A avaliação foi feita de forma independente e isolada pelos dois investigadores, tendo construído uma grelha para comparação e discussão.

\section{Extração de dados}

Recolhemos os dados referentes ao maior tempo de follow-up comparável entre os estudos e relativos ao mesmo escalão etário. Por se tratar de um estudo de demonstração de superioridade (vacinas vs controlo), optámos por extrair apenas os resultados segundo o princípio de intenção de tratar, isto é, incluindo toda a população randomizada, independentemente de desistências, perdas ou não adesão ao protocolo, de forma a minimizar a hipótese de haver viés de atrito ou erro tipo I. A extração de dados foi feita de forma independente e isolada pelos dois investigadores, tendo construído uma tabela para comparação e discussão.

\section{Síntese dos resultados obtidos e medidas de efeito}

A síntese dos resultados obtidos foi feita através de uma análise quantitativa comparando os dados segundo o princípio de intenção de tratar. $\mathrm{Na}$ análise estatística aplicámos o modelo estatístico de efeitos aleatórios por não se tratar da aplicação da mesma vacina (bivalente versus tetravalente); usámos o risco relativo como medida do efeito e os valores de $\chi^{2}$ e de $\mathrm{I}^{2}$ como medidas de heterogeneidade; usámos o software RevMan $5.3^{26}$ para a realização da análise quantitativa.

\section{Descrição formal de confundidores entre os estudos selecionados}

Considerámos que poderia constituir um confundidor do efeito cumulativo o facto de terem sido usadas vacinas diferentes nos estudos, no entanto, de forma a minimizar o efeito deste possível confundidor optámos por usar na análise um modelo estatístico que o tivesse em conta.

\section{Avaliação da qualidade da evidência}

Avaliámos a qualidade da evidência usando o sistema GRADE ${ }^{27}$ Este sistema tem em consideração cinco critérios - design/metodologia, consistência, objetividade, precisão e viés de publicação - permitindo classificar a qualidade da evidência em quatro níveis - alta, moderada, baixa e muito baixa.

A avaliação foi realizada pelos investigadores de forma isolada e independente, tendo construído uma grelha para comparação e discussão.

\section{RESULTADOS \\ Seleção dos estudos}

Da estratégia de pesquisa e seleção de estudos (Fig. 1) resultaram inicialmente 417 citações que foram avaliadas para elegibilidade para este estudo, tendo resultado em dois RCTs incluídos na análise qualitativa e na análise quantitativa.

\section{Características do estudo}

Os RCTs selecionados envolveram um total de 9571 participantes (todos do sexo feminino): 4792 mulheres no grupo vacinado contra o HPV e 4779 mulheres no grupo controlo. Ambos os ensaios reportam dados relativos a um período de follow-up mínimo de quatro anos, sendo que o estudo VIVIANE ${ }^{13}$ reporta resultados intercalares e encontra-se ainda a decorrer com o objetivo de realizar um follow-up mínimo de sete anos após a vacinação, enquanto o estudo $X$ Castellsagué et $\mathrm{al}^{28}$ reporta resultados finais de um ensaio clínico com o objetivo de efetuar um follow-up de quatro anos. A idade média dos participantes no início do estudo VIVANE foi de aproximadamente 37 anos, enquanto no estudo $X$ Castellsagué et al foi cerca de 34 anos; no primeiro foi feita uma estratificação por idade em três grupos, ao passo que no segundo foi feita uma estratificação em apenas dois grupos. Nos estudos não participaram mulheres grávidas. $\mathrm{O}$ estudo VIVIANE avalia a eficácia, segurança e imunogenicidade da vacina bivalente enquanto o estudo X Castellsagué et al avaliou os mesmos parâmetros relativos à vacina tetravalente. Em ambos os estudos foi 


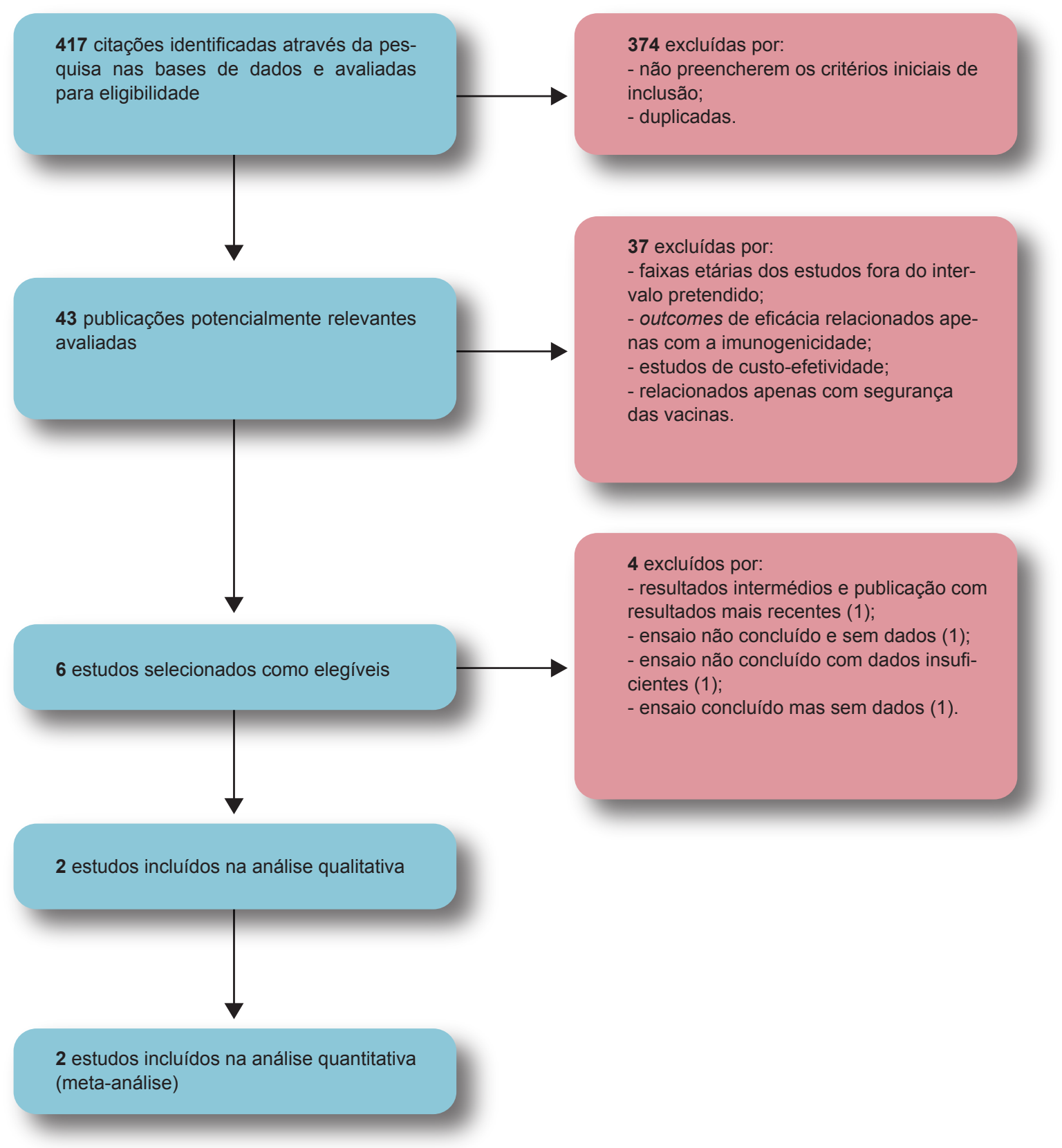

Figura 1 - Fluxograma de pesquisa e seleção de estudos

definido como endpoint primário para a avaliação da eficácia da vacina a infeção persistente por HPV 16 e/ou 18, e/ou a presença de lesões do colo do útero provocadas pela infeção persistente, no estudo $X$ Castellsagué et al foi ainda acrescentada a presença de doença genital externa associada ao HPV 16 e/ou 18; ambos avaliaram também a segurança e tolerabilidade das vacinas. Os dois estudos foram desenhados e patrocinados pelos fabricantes das vacinas, e foram conduzidos num total de 16 países.

A Tabela 3 sumariza as principais características dos dois RCTs.

\section{Risco de viés dos estudos}

O risco de presença de viés dos estudos foi avaliado como sendo baixo para ambos os estudos. A Fig. 2 mostra a avaliação do risco de presença de viés segundo a ferramenta de avaliação da presença de viés da Cochrane. ${ }^{25}$

\section{Síntese de resultados}

A Fig. 3 mostra os riscos relativos (RRs) individuais assim como o RR combinado dos RCTs com intervalos de confiança de $95 \%(95 \% \mathrm{Cls})$ referente ao período de follow-up de 48 meses. O RR combinado do outcome infeção persistente ( $\geq$ seis meses) e/ou lesão provocada pela infeção persistente por HPV 16 e/ou 18 (CIN1 ou +) foi de 0,59 (95\% Cl: 0,50 a 0,71), correspondendo a uma redução do RR de $41 \%$ (95\% Cl: 29 a 50\%) para as mulheres vacinadas. A heterogeneidade foi nula $\left(\chi^{2}=0.00 ; r^{2}=0 \%\right)$. 
Tabela 3 - Resumo das principais características dos RCTs

\begin{tabular}{|c|c|c|}
\hline Estudo & VIVIANE $^{13}$ & $\mathrm{X}$ Castellsagué et $\mathrm{al}^{28}$ \\
\hline Vacina / Controlo & Bivalente / Placebo & Tetravalente / Placebo \\
\hline $\mathrm{N}^{\circ}$ de participantes & $\begin{array}{l}\text { Total }=5752 \\
\text { Vacina }=2881 / \\
\text { Controlo }=2871\end{array}$ & $\begin{array}{l}\text { Total = } 3819 \\
\text { Vacina = } 1911 / \\
\text { Controlo }=1908\end{array}$ \\
\hline Follow-up & 48 Meses (a decorrer) & 48 Meses (concluído) \\
\hline Escalão etário & $\begin{array}{l}>25 \text { anos } \\
\text { Três grupos: } \\
{[26-35] \text { anos; }} \\
{[36-45] \text { anos; }} \\
{[\geq 46] \text { anos }}\end{array}$ & $\begin{array}{l}{[24-45] \text { anos }} \\
\text { Dois grupos: } \\
{[24-34] \text { anos; }} \\
{[35-45] \text { anos }}\end{array}$ \\
\hline Idade média no início do estudo & $37,0 \pm 7,2$ anos & $34,3 \pm 6,3$ anos \\
\hline \multirow[t]{2}{*}{$\begin{array}{l}\text { População para análise de eficácia } \\
\text { segundo o princípio de intenção de tratar }\end{array}$} & $\begin{array}{l}\text { Todos os participantes do estudo que } \\
\text { receberam pelo menos uma dose da } \\
\text { vacina/placebo e fizeram pelo menos uma } \\
\text { visita de follow-up; incluiu um subgrupo } \\
\text { de mulheres com história de infeção ou } \\
\text { doença anterior provocada por HPV }=15 \% \\
\text { dos participantes; endpoints avaliados } \\
\text { independentemente da presença de DNA } \\
\text { do HPV ou do serostatus no início do } \\
\text { estudo. }\end{array}$ & $\begin{array}{l}\text { Todos os participantes do estudo que } \\
\text { receberam pelo menos uma dose da } \\
\text { vacina/placebo e fizeram pelo menos uma } \\
\text { visita de follow-up; incluiu mulheres que } \\
\text { eram, no ponto inicial, seropositivas e/ } \\
\text { ou PCR positivas para genótipos do HPV } \\
\text { da vacina ou não-vacinais; endpoints } \\
\text { avaliados independentemente da presença } \\
\text { de DNA do HPV ou do serostatus no início } \\
\text { do estudo. }\end{array}$ \\
\hline & $\begin{array}{l}n(\text { Total): } \\
\text { Vacina = } 2772([26-35] \text { anos = } 1225 \\
{[36-45] \text { anos = } 1245 ;[\geq 46] \text { anos = 300); }} \\
\text { Controlo = } 2779([26-35] \text { anos = } 1243 \\
{[36-45] \text { anos = } 1229 ;[\geq 46] \text { anos = 307) }}\end{array}$ & $\begin{array}{l}n(\text { Total }): \\
\text { Vacina }=1886([24-34] \text { anos }=937 ; \\
[35-45] \text { anos }=949) ; \\
\text { Controlo = } 1883([24-34] \text { anos }=944 ; \\
[35-45] \text { anos }=939)\end{array}$ \\
\hline \multirow[t]{2}{*}{ Endpoints primários } & $\begin{array}{l}\text { Infeção persistente } \geq \text { seis meses por HPV } \\
16 \text { e/ou 18; } \\
\text { Neoplasia intra-epitelial cervical - CIN1 ou } \\
\text { superior relacionada com HPV } 16 \text { e/ou } 18\end{array}$ & $\begin{array}{l}\text { Infeção persistente } \geq \text { seis meses por HPV } \\
16 \text { e/ou } 18 ; \\
\text { Neoplasia intra-epitelial cervical - CIN1 ou } \\
\text { superior relacionada com HPV } 16 \text { e/ou } 18 \text {; } \\
\text { Doença genital externa relacionada com } \\
\text { HPV } 16 \text { ou } 18\end{array}$ \\
\hline & $\begin{array}{l}\text { Casos (Events): } \\
\text { Vacina = } 90([26-35] \text { anos = 63; }[36-45] \\
\text { anos = 23; }[\geq 46] \text { anos = 4); } \\
\text { Controlo = } 158([26-35] \text { anos }=97 ;[36-45] \\
\text { anos = 48; }[\geq 46] \text { anos = 13) }\end{array}$ & $\begin{array}{l}\text { Casos }(\text { Events): } \\
\text { Vacina = } 95([24-34] \text { anos }=60 ;[35-45] \\
\text { anos }=35) ; \\
\text { Controlo }=160([24-34] \text { anos }=100 ;[35-45] \\
\text { anos }=60)\end{array}$ \\
\hline Países & $\begin{array}{l}\text { Austrália; } \\
\text { Canadá; } \\
\text { E.U.A; } \\
\text { Filipinas; } \\
\text { Holanda; } \\
\text { México; } \\
\text { Perú; } \\
\text { Portugal; } \\
\text { Reino Unido; } \\
\text { Rússia; } \\
\text { Singapura; } \\
\text { Tailândia }\end{array}$ & $\begin{array}{l}\text { Alemanha; } \\
\text { Colômbia; } \\
\text { Espanha; } \\
\text { E.U.A; } \\
\text { Filipinas; } \\
\text { França; } \\
\text { Tailândia }\end{array}$ \\
\hline
\end{tabular}

Para a realização da análise quantitativa foram excluídos os resultados do grupo [ $\geq 46]$ anos, relativo ao estudo VIVIANE, de forma a se poder fazer uma comparação o mais equiparável possível entre os estudos. 


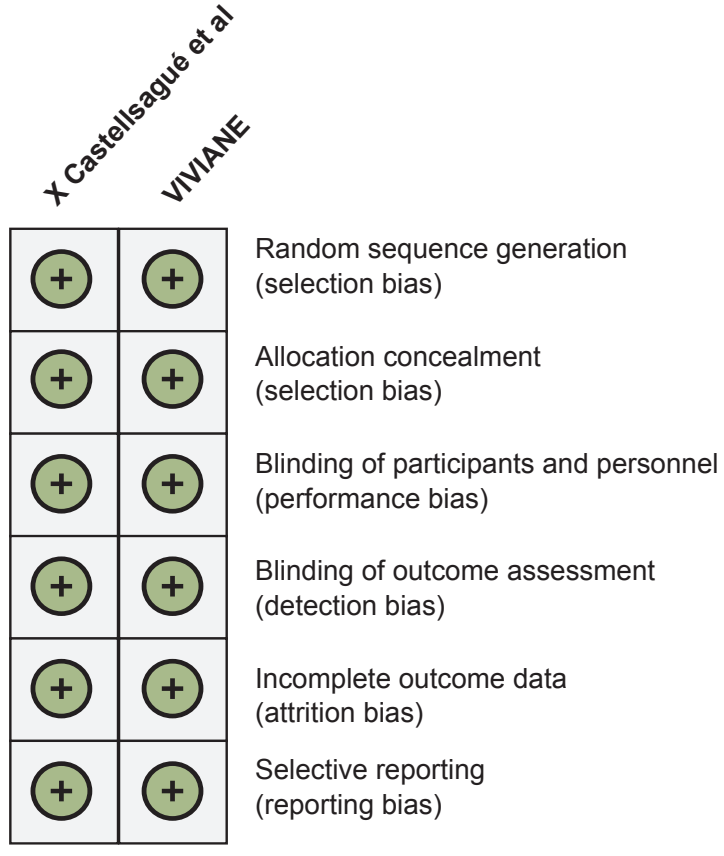

Low risk of bias

High risk of bias ? Unclear risk of bias

Figura 2 - Avaliação da presença de risco de viés
A segurança e tolerabilidade das vacinas foi semelhante nos grupos vacina e nos grupos controlo dos estudos (incidência de eventos adversos sérios, novas doenças crónicas ou despoletar de doenças autoimunes sem diferenças estatisticamente significativas), com a exceção de um ligeiro aumento da reação local no sítio da injeção (dor, edema e eritema) no grupo vacina em ambos os estudos.

\section{Avaliação da qualidade da evidência}

A qualidade da evidência foi classificada como "alta", uma vez que: se trata de RCTs; o risco de presença de viés foi considerado baixo; e não foram encontradas lacunas relacionadas com as outras áreas definidas nos critérios GRADE - consistência; objetividade; precisão; ou viés de publicação.

\section{DISCUSSÃO}

O resultado da agregação estatística realizada com os dados da evidência atual reforça os resultados dos estudos disponíveis e demonstra que a administração das vacinas contra o HPV a mulheres adultas com idade compreendia entre os 24 e os 45 anos resulta numa redução importante do risco de adquirir infeção persistente pelo HPV 16 e/ou 18 e/ou adquirir lesões precursoras de CCU causadas pela infeção persistente, o que advoga os benefícios da vacinação neste grupo etário. Ambas as vacinas parecem apresentar benefício semelhante.

Os estudos incluídos neste trabalho definiram a infeção persistente com duração de pelo menos seis meses e a

\begin{tabular}{|c|c|c|c|c|c|c|c|}
\hline \multirow[b]{2}{*}{ Study or subgroup } & \multicolumn{2}{|c|}{ Vacine } & \multicolumn{2}{|c|}{ Control } & \multicolumn{3}{|c|}{ Risk ratio } \\
\hline & Events & Total & Events & Control & Weight & M - H, Random, $95 \% \mathrm{Cl}$ & Year \\
\hline X Castellsagué et al & 95 & 1886 & 160 & 1883 & $53.0 \%$ & $0.59[0.46,0.76]$ & 2011 \\
\hline VIVIANE & 86 & 2472 & 145 & 2472 & $47.0 \%$ & $0.59[0.46,0.77]$ & 2014 \\
\hline Total $(95 \% \mathrm{Cl})$ & & 4358 & & 4355 & $100.0 \%$ & $0.59[0.50,0.71]$ & \\
\hline Total events & 181 & & 305 & & & & \\
\hline \multicolumn{8}{|c|}{ Heterogeneity: $\mathrm{Tau}^{2}=0.00 ; \mathrm{Chi}^{2}=0.00, \mathrm{df}=1\left(p=1.00 ; \mathrm{I}^{2}=0 \%\right)$} \\
\hline \multicolumn{8}{|c|}{ Test for overall effect: $Z=5.73(p<0.00001)$} \\
\hline
\end{tabular}

\section{Risk ratio}

Study or subgroup M - H, Random, $95 \% \mathrm{Cl}$

$X$ Castellsagué et al VIVIANE

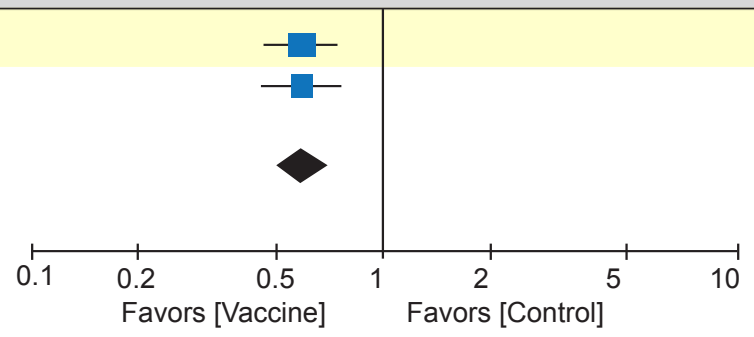

Figura 3 - Forest plot: Eficácia da vacinação contra o HPV na prevenção do CCU nas mulheres com idade no intervalo [24 - 45] anos segundo o princípio de intenção de tratar; outcome: Infeção persistente ( $\geq$ seis meses) e/ou lesão provocada pela infeção persistente por HPV 16 e/ou 18 (CIN1 ou +); follow-up: 48 meses. 
lesão CIN1 ou superior como outcomes para avaliação da eficácia das vacinas na prevenção do CCU. Embora se recomende avaliar a persistência da infeção 12 meses após a sua deteção para identificação de mulheres com risco aumentado de adquirir lesões precursoras de $\mathrm{CCU}^{29}$ e se considere a lesão CIN1 como uma lesão intra-epitelial de baixo grau com baixo potencial de progressão, ${ }^{30,31}$ a assunção efetuada pode considerar-se válida por tratar-se de ensaios cujas participantes são mulheres adultas e de se verificar que com o aumento da idade ocorre um declínio da função imunitária e consequente aumento do risco de aquisição de infeção persistente e de lesões precursoras do CCU.9,15

Ainda que a maior taxa de incidência de infeção se verifique pouco tempo após o início da atividade sexual, alguns estudos têm demonstrado uma distribuição bimodal da prevalência de DNA dos HPV de alto risco, ocorrendo o segundo pico nas mulheres na quarta e quinta décadas de vida, ${ }^{32,33}$ o que, conjuntamente com o facto de a resolução natural da infeção não conferir imunidade e de o CCU ocorrer em qualquer idade, reforça a importância do papel da vacinação contra o HPV nas mulheres adultas.

Apesar da importante eficácia das vacinas contra o HPV nas mulheres entre os 24 e 45 anos, deve evitar-se a falsa sensação de proteção total na mulher vacinada, uma vez que, a eficácia demostrada na redução do risco de adquirir infeção persistente e/ou adquirir lesões precursoras de CCU causadas pela infeção persistente não foi total $(41 \%)$, e não existe ainda uma vacina capaz de, comprovadamente, conferir proteção contra todos os genótipos oncogénicos. Não é recomendável descurar o papel do rastreio na prevenção do CCU, mesmo na mulher vacinada.

A otimização de uma estratégia de vacinação em articulação com o rastreio do CCU poderá vir a favorecer a redução do número de casos e mortes relacionadas com o HPV.

A segurança das vacinas não revelou diferenças em relação ao controlo, o que está de acordo com os estudos de segurança e com as orientações da Organização Mundial de Saúde. ${ }^{16}$

\section{Limitações}

Esta análise apresenta como limitações não ter sido possível avaliar e comparar a eficácia da vacinação nas mulheres com mais de 45 anos, por apenas um dos estudos incluir um pequeno grupo de mulheres nessa faixa etária; nem ter sido possível comparar as diferenças entre os subgrupos etários estratificados nos estudos, por não coincidirem nos limites. O facto de existirem poucos estudos que avaliam a questão da vacinação nas mulheres com mais de 24 anos representa também uma limita- ção, sendo que, no entanto, se encontram a decorrer três ensaios registados em ClinicalTrials.gov (NCT00090220; NCT00693966; e NCT01735006) - um dos quais já concluído, mas ainda sem dados dos resultados - que poderão vir a reforçar a robustez dos resultados desta análise. São pontos fortes, o facto de ambos os estudos incluídos representarem estudos multinacionais e o facto de se ter realizado uma análise segundo o princípio de intenção de tratar, uma vez que se trata de demonstrar superioridade das vacinas em comparação com controlo.

Existe a necessidade de se realizar mais estudos que permitam avaliar a eficácia da vacinação na mulher adulta e de que sejam incluídas participantes com idade superior a 45 anos. É importante estudar-se a otimização de estratégias de vacinação em articulação com o rastreio de forma a melhorar a prevenção do CCU e devem realizar-se estudos de custo-efetividade que avaliem o impacto dessas estratégias no desenvolvimento de novas políticas de vacinação e rastreio. Para além de se demonstrar a eficácia das vacinas, é ainda importante realizar estudos que permitam comparar a eficácia das diferentes vacinas na mulher adulta, incluindo também a vacina mais recente (nonavalente) nos estudos.

\section{CONCLUSÃO}

A evidência atual demostra que a administração das vacinas contra o HPV a mulheres adultas, pelo menos até aos 45 anos de idade, apresenta uma eficácia importante na prevenção do risco de desenvolvimento de CCU; as muIheres adultas não vacinadas devem ser informadas acerca deste benefício. Contudo, a redução do risco de desenvolver infeção persistente pelo HPV 16 e/ou 18 e/ou adquirir lesões precursoras de CCU causadas pela infeção persistente não é total, devendo por isso evitar-se a falsa sensação de proteção total na mulher vacinada e evitar descurar o papel do rastreio na prevenção do CCU.

\section{AGRADECIMENTOS}

Agradecemos a António Rodrigues, médico especialista de Medicina Geral e Familiar na USF Corgo, pela sua contribuição na revisão do texto do artigo.

\section{CONFLITO DE INTERESSES}

Os autores declaram não ter nenhum conflito de interesses relativamente ao presente artigo.

\section{FONTES DE FINANCIAMENTO}

Não existiram fontes externas de financiamento para a realização deste artigo.

\section{REFERÊNCIAS}

1. Fitzmaurice C, Dicker D, Pain A, Hamavid H, Moradi-Lakeh M, Maclntyre MF, et al. The Global Burden of Cancer 2013. JAMA Oncol. 2015;1:50527.

2. Bruni L, Barrionuevo-Rosas L, Albero G, Aldea M, Serrano B, Valencia

S, et al. Human Papillomavirus and Related Diseases in the World. Summary Report 2015-04-08. . Barcelona: ICO Information Centre on HPV and Cancer (HPV Information Centre); 2015.

3. IARC IAfRoC-. World Cancer Report 2014. Lyon: World Health 
Organization; 2014

4. Bruni L, Barrionuevo-Rosas L, Albero G, Aldea M, Serrano B, Valencia $S$, et al. Human Papillomavirus and Related Diseases in Portugal. Summary Report 2015-03-20. Barcelona: ICO Information Centre on HPV and Cancer (HPV Information Centre); 2015.

5. Direcção Geral da Saúde. A vacinação contra o vírus do papiloma humano (HPV) em Portugal. Boletim Vacinação - edição especial 2014;8:1-4.

6. Kumar S, Biswas M, Jose T. HPV vaccine: Current status and future directions. Med J Armed Forces India. 2015;71:171-7.

7. Schiffman M, Castle PE, Jeronimo J, Rodriguez AC, Wacholder S. Human papillomavirus and cervical cancer. Lancet 2007;370:890-907.

8. Walboomers JM, Jacobs MV, Manos MM, Bosch FX, Kummer JA, Shah $\mathrm{KV}$, et al. Human papillomavirus is a necessary cause of invasive cervical cancer worldwide. J Pathol. 1999;189:12-9.

9. Ginecologia SPd. Vacinas contra o HPV Reunião de Consenso Nacional - Cascais:RCN; 2010.

10. Brisson M, Laprise JF, Drolet M, Van de Velde N, Franco EL, Kliewer EV, et al. Comparative cost-effectiveness of the quadrivalent and bivalent human papillomavirus vaccines: a transmission-dynamic modeling study. Vaccine. 2013;31:3863-71.

11. Erickson BK, Landers EE, Huh WK. Update on vaccination clinical trials for HPV-related disease. Clin Ther. 2014;36:8-16.

12. Leite I, Lisboa C, Azevedo F. Vírus do papiloma humano e vacinação. Rev Port Dermatol Venereol. 2011;69:305-402.

13. Skinner SR, Szarewski A, Romanowski B, Garland SM, Lazcano-Ponce $E$, Salmerón J, et al. Efficacy, safety, and immunogenicity of the human papillomavirus 16/18 AS04-adjuvanted vaccine in women older than 25 years: 4-year interim follow-up of the phase 3, double-blind, randomised controlled VIVIANE study. Lancet. 2014;384:2213-27.

14. Van de Velde N, Boily MC, Drolet M, Franco EL, Mayrand MH, Kliewer $\mathrm{EV}$, et al. Population-level impact of the bivalent, quadrivalent, and nonavalent human papillomavirus vaccines: a model-based analysis. J Natl Cancer Inst. 2012;104:1712-23.

15. Castellsague $X$, Schneider A, Kaufmann AM, Bosch FX. HPV vaccination against cervical cancer in women above 25 years of age: key considerations and current perspectives. Gynecol Oncol. 2009;115:S15-23.

16. De Vincenzo R, Conte C, Ricci C, Scambia G, Capelli G. Long-term efficacy and safety of human papillomavirus vaccination. Int J Womens Health. 2014;6:999-1010.

17. Westra TA, Rozenbaum MH, Rogoza RM, Nijman HW, Daemen T, Postma MJ, et al. Until which age should women be vaccinated against HPV infection? Recommendation based on cost-effectiveness analyses. J Infect Dis. 2011;204:377-84.

18. Carozzi F, De Marco L, Gillio-Tos A, Del Mistro A, Girlando S, Baboci L, et al. Age and geographic variability of human papillomavirus highrisk genotype distribution in a large unvaccinated population and of vaccination impact on HPV prevalence. J Clin Virol. 2014;60:257-63.
19. Mazza D, Petrovic K, Grech C, Harris N. HPV vaccination in women aged 27 to 45 years: what do general practitioners think? BMC Women's Health. 2014;14:91

20. Delere Y, Wichmann O, Klug SJ, Wichmann O, Klug SJ, van der Sande $M$, et al. The efficacy and duration of vaccine protection against human papillomavirus: a systematic review and meta-analysis. Dtsch Arztebl Int. 2014;111:584-91.

21. FDA. [consultado 2015 ago 07]. Disponível em: http://www.fda.gov/ newsevents/newsroom/pressannouncements/ucm426485.htm. 2014.

22. EMA. [consultado 2015 ago 07]. Disponível em: http://www.ema.europa. eu/docs/en_GB/document_library/EPAR_-_Summary_for_the_public/ human/003852/WC500189114.pdf. 2015.

23. Castle PE, Schmeler KM. HPV vaccination: for women of all ages? Lancet. 2014;384:2178-9.

24. Moher D, Liberati A, Tetzlaff J, Altman DG, Group P. Preferred reporting items for systematic reviews and meta-analyses: the PRISMA statement. PLoS Med. 2009;6:e1000097.

25. Higgins JP, Altman DG, Gøtzsche PC, Jüni P, Moher D, Oxman AD, et al. The Cochrane Collaboration's tool for assessing risk of bias in randomised trials. BMJ. 2011;343:d5928.

26. Review Manager (RevMan) [Computer program]. Version 5.3 Copenhagen: The Nordic Cochrane Centre, The Cochrane Collaboration. 2014

27. Balshem $H$, Helfand M, Schunemann HJ, Oxman AD, Kunz R, Brozek $\mathrm{J}$, et al. GRADE guidelines: 3. Rating the quality of evidence. J Clin Epidemiol. 2011;64:401-6.

28. Castellsague X, Munoz N, Pitisuttithum P, Ferris D, Monsonego J, Ault K et al. End-of-study safety, immunogenicity, and efficacy of quadrivalent HPV (types $6,11,16,18$ ) recombinant vaccine in adult women 24-45 years of age. Br J Cancer. 2011;105:28-37.

29. Rositch AF, Koshiol J, Hudgens MG, Razzaghi H, Backes DM, Pimenta $\mathrm{JM}$, et al. Patterns of persistent genital human papillomavirus infection among women worldwide: a literature review and meta-analysis. Int $J$ Cancer. 2013;133:1271-85

30. Petry KU. Management options for cervical intraepithelial neoplasia. Best Pract Res Clin Obstet Gynaecol. 2011;25:641-51.

31. Howell LP, Zhou H, Wu W, Davis R. Significance of subclassifying high-grade squamous intraepithelial lesions into moderate dysplasia/ CIN II versus severe dysplasia/CIN III/CIS in the Bethesda System terminology. Diagn Cytopathol. 2004;30:362-6.

32. de Sanjose S, Diaz M, Castellsague X, Clifford G, Bruni L, Muñoz N, et al. Worldwide prevalence and genotype distribution of cervical human papillomavirus DNA in women with normal cytology: a meta-analysis. Lancet Infect Dis. 2007;7:453-9.

33. Munoz N, Mendez F, Posso H, Molano M, van den Brule AJ, Ronderos $M$, et al. Incidence, duration, and determinants of cervical human papillomavirus infection in a cohort of Colombian women with normal cytological results. J Infect Dis. 2004;190:2077-87. 